\title{
HISTORIA RECIENTE DE LA UNIVERSIDAD DE ALCALÁ (Segunda parte: Primer intento de resurrección)
}

\section{RECENT HISTORY OF THE UNIVERSITY OF ALCALA \\ (Part Two: First attempt at resurrection)}

\section{AUTORES}

José Morilla Critz: Profesor en la Facultad de Ciencias Económicas y Empresariales. Universidad de Alcalá.

Catedrático de Universidad. Área de Fundamentos de la Economía. jose.morilla@aol.com

\section{RESUMEN}

En esta ocasión, José Morilla nos cuenta los esfuerzos de la sociedad alcalaína por conseguir que, la Universidad Autónoma de Madrid, creada por Decreto-Ley de 6 de junio de 1968, tuviera su ubicación en la ciudad Complutense. Mientras se movilizaba la ciudadanía en una lucha desigual contra las fuerzas vivas del régimen franquista, las clases de la nueva universidad empezaron a ser impartidas en distintas dependencias, totalmente ajenas al mundo universitario, de la capital del Estado, como fue el Pabellón de Cristal de la Casa de Campo.

\section{PALABRAS CLAVE}

Historia - Universidad de Alcalá - Renacimiento 


\begin{abstract}
On this occasion, José Morilla recounts the efforts of society by getting Alcala, Universidad Autonoma de Madrid, created by Decree Law of June 6, 1968, had its location in the city Complutense. While mobilizing citizens in an unequal struggle against the forces of the Franco regime, the new university classes began to be taught in different departments, completely outside the academic world, the state capital, as was the Crystal Pavilion Casa de Campo.
\end{abstract}

\title{
KEY WORDS
}

History - University of Alcalá - Renaissance

En esta ocasión, José Morilla nos cuenta los esfuerzos de la sociedad alcalaína por conseguir que, la Universidad Autónoma de Madrid, creada por Decreto-Ley de 6 de junio de 1968, tuviera su ubicación en la ciudad Complutense. Mientras se movilizaba la ciudadanía en una lucha desigual contra las fuerzas vivas del régimen franquista, las clases de la nueva universidad empezaron a ser impartidas en distintas dependencias, totalmente ajenas al mundo universitario, de la capital del Estado, como fue el Pabellón de Cristal de la Casa de Campo.

\section{El Renacimiento de la Universidad de Alcalá (Por José Morilla Critz. Catedrático de Universidad. Area de Fundamentos de la Economía)}

Segunda parte: Primer intento de resurrección. 
4. Los años sesenta: desarrollo industrial y proyecto universitario frustrado en Alcalá.

La cercanía de Alcalá de Henares a Madrid, había sido en el siglo XIX tal vez el principal motivo de su desmantelamiento como ciudad universitaria. Igualmente había sido la razón del emplazamiento en ella de unidades militares y de instalaciones carcelarias. Estos componentes tuvieron como virtud principal para el futuro, aunque no fuera buscada en su origen, mantener en pie, ya fuera semirruinosos, buena parte de sus edificios histórico-universitarios.

Pero a finales de los años cincuenta del siglo XX, esa cercanía empezó a producir la entrada de la ciudad complutense en el circuito industrial que la urbe madrileña vio crecer rápidamente en su entorno, tras el "Plan de Estabilización" de 1959 y la apertura de la economía española al exterior.

Después de la Guerra Civil Alcalá tenía una población de 20.335 habitantes, de los cuales 4.424 eran presos y reclusos y, del resto,, el 20 por 100 era población militarNota2. La estructura de su economía, aparte del sector comercial potenciado, en parte, por la población castrense, era básicamente agrícola: una gran parte en forma de secano y otra de un regadío en expansión al mercado madrileño. Sin embargo, desde principios de los años sesenta la ciudad experimentó un extraordinario cambio económico que llevó aparejada en quince años su transformación demográfica, ésta, un gran crecimiento de la actividad constructora y, de aquí, una desfiguración radical.

En 1975 Alcalá de Henares tenía ya una población de hecho de 104.000 habitantes (100.300 de derecho), de la que el 69 por 100 era población inmigrada de Castilla - La Mancha, Extremadura, y AndalucíaNota3, habiendo llegado la mayor parte de ella en el periodo 1965-1974. 
El crecimiento había sido la consecuencia inmediata de la instalación industrial, habiendo pasado el número de establecimientos de este tipo de 132 en 1962 a 402 en 1974, siendo los de la primera fecha fundamentalmente pequeños y englobándose en la segunda grandes factorías de electrodomésticos, textiles industriales, construcción y sector químico-farmacéutico.

El impacto en la construcción de este crecimiento demográfico queda palpable al considerar que el censo de viviendas entre 1960 y 1975 aumentó en un 358,37 por 100Nota4. La desfiguración urbanística se tradujo, fundamentalmente, en la construcción de todo un cinturón de bloques de pisos de no muy buena calidad en torno a la tradicional ciudad, destruyendo, por una parte, una buena porción de los campos de regadío y, por otra, las zonas de hábitat residencial de finales del XIX y principios del XX. El llamado "centro histórico" fue poco modificado debido a que en 1968 fue declarado "conjunto histórico" y, por tanto, sometido a unas normas especiales de construcción en él.

En ese casco histórico se situaba la totalidad de los antiguos edificios universitarios que, bien en ruinas o en manos del Ejercito, del Ministerio de Justicia, de otros organismos públicos, o de particulares, lograron salvarse en su mayoría. Pero sobre todo, a pesar de la desfiguración urbanística general, quedó rodeada por las nuevas edificaciones la trama urbana renacentista (aunque las viviendas particulares fueran la mayoría del siglo pasado). Mas en cuanto a hábitat, ese centro se fue despoblando a medida que la nueva fisionomía industrial de la ciudad se consolidaba.

A corto plazo, por tanto, la historia universitaria de Alcalá, incluso en sus vestigios más materiales, parecía que sucumbía en Alcalá ya definitivamente ante un presente industrial. Y sin embargo, a mediados de los años sesenta, factores que nada tenían que ver con la tradición histórica, sino, precisamente, con ese desarrollo industrial de 
la capital de España, dieron una nueva oportunidad para que los alcalaínos vislumbraran la posibilidad de recuperar el carácter universitario de la ciudad.

Crecimiento demográfico y crecimiento económico empezaron a producir incremento de la población universitaria en las grandes ciudades industriales, especialmente en Madrid, Barcelona y Bilbao: El nuevo Ministerio de Educación y Ciencia, en coherencia con lo que iba a ser el intento de planificación económica plasmado en el Primer Plan de Desarrollo, elaboró una nueva "Ley General de Educación" que, en el aspecto universitario, preveía la posibilidad de creación de nuevas universidades. Con tal medida se intentaba superar el encorsetado marco de los doce distritos universitarios existentes hasta entonces y se pretendían ensayar algunas nuevas formas de organización de la enseñanza universitaria.

Con esa finalidad, el Ministro de Educación anunció en 1965, en un discurso en Cuenca, la necesidad de descongestionar las ya entonces masificadas universidades de Madrid y Barcelona y otras del país, mediante la creación de varias de ellas. Estas intenciones se concretarían más adelante en la propuesta de creación, dentro del II Plan de Desarrollo, de las nuevas universidades de Madrid, Barcelona y Bilbao y la concertación, con diversas administraciones locales y privadas, de la creación de colegios universitarios en otras localidades.

Esta nueva actitud del Estado era lógico que provocara en Alcalá de Henares un inmediato efecto, de tal manera que tras la primera declaración ministerial en aquel sentido (mayo de 1965), en el Pleno del Ayuntamiento de Alcalá se aprobó un ruego del concejal don Francisco Javier García Gutiérrez, presidente de la Comisión de Cultura, de solicitar al Ministerio de Educación y Ciencia que la "descongestión" universitaria de Madrid se hiciera en Alcalá. Surgió así una línea de interacción ciudad - gobierno central en torno a la creación de una nueva Universidad en Alcalá, que veremos actuar hasta 1977. 
Junto a esta línea de interacción, también en las mismas fechas comenzó a manifestarse un creciente interés entre los representantes de la Universidad Central de Madrid por un asentamiento en la ciudad de la que había surgido aquella misma universidad en el siglo anterior. Es difícil deslindar en ese interés lo que había de apreciación erudita por la "recuperación de señas de identidad" que pueda ser considerado un signo de evolución de la sensibilidad ligada al propio desarrollo económico, de lo que era el agobio que se empezaba a manifestar ante la masificación (lo que puede tener también una lectura "conservadora", puesto que la masificación se manifestaba en la Central en una subversión creciente, que ponía en cuestión la otrora tranquila situación de la jerarquía universitaria y de los grupos de poder más tradicionales). En marzo de 1966 el entonces rector de la Universidad Central, profesor Gutiérrez Ríos, expresó en Alcalá que:

Es nuestro propósito dejar aquí un testimonio permanente de homenaje y gratitud de la Universidad de Alcalá por la conservación de estos edificios.

Y en 1967, se conoció un proyecto de la Universidad Central de "recrear" en Alcalá estudios de Filosofía y Letras, concretamente de Latín, Griego y Hebreo, en el Colegio de San Jerónimo, o patio Trilingüe.

De la línea proveniente de los proyectos del Gobierno, las características del emplazamiento que se buscaba para una nueva universidad en Madrid derivaban del siguiente programa: a la nueva universidad se la quería hacer nacer como "ciudad universitaria completa" emulando la construida como tal en los tiempos de Alfonso XIII; por otra parte se pretendía introducir un modelo más o menos estadounidense de estructura física (edificios integrados todos en un campus común, con instalaciones auxiliares) y académica (departamentos interfacultativos y primacía de profesorado contratado), Finalmente, había que resolver también el grave problema 
de orden público y político que provocaba, dentro de un régimen político como aquél, una población universitaria revolucionaria en pleno corazón de Madrid.

Un proyecto como ése, para el que se adoptó el nombre de "Universidad Autónoma", con el fin de deslindarla de lo que se entendían distritos universitarios hasta entonces y, también, conseguir el efecto propagandístico de anunciar que se estaba protagonizando un cierto reformismo no centralista, con una cierta autonomía de funcionamiento interno, necesitaba un extenso territorio a obtener por el Ministerio de Educación y Ciencia lo más rápida y favorablemente posible en las cercanías de Madrid.

El carácter de núcleo militar que había tenido Alcalá desde hacía tiempo, jugó inmediatamente como baza a su favor, puesto que, junto a la ciudad, el Ejército del Aire disponía de una antigua base aérea, ahora casi en desuso, de 240 hectáreas de extensión, con algunas instalaciones de infraestructura y circulación interna que podrían inicialmente ser útiles: además por medio de ellas cruzaba la línea ferroviaria Madrid-Barcelona, con trenes de cercanías hasta Guadalajara.

Las razones históricas también parecían actuar a favor de Alcalá. Éstas eran un argumento en boca de instituciones y personas alcalaínas, y también lo fueron de algunos medios de comunicación nacionales $y$, sobre todo, de algunas personalidades muy definidas.

Pero la lógica (mucho más lógica vistas las cosas en perspectiva) no es habitual que se abra paso. En torno a tan ambicioso proyecto, en un momento de expansión urbana de la capital de España, hemos de comprender que se crearan grandes expectativas. Las expectativas especuladoras podrán ser una razón que no jugara a favor de la disponibilidad, precisamente, de unos terrenos públicos, si entendemos 
que el poder, y menos aún el de un gobierno no democrático ni controlado por la opinión pública, no es ajeno a los grupos de presión privados.

Las expectativas, en todo caso, no tenían que ser necesariamente de tipo especulativo e irregular. Como era de esperar, tanto Madrid como diversos municipios colindantes, incluso Guadalajara, elevaron al Gobierno su deseo de verse "agraciados" con la sede de la nueva universidad. Pero nada fue comparable al eco que adquirió en la prensa nacional la posibilidad de aprovechar la ocasión para recuperar la Universidad de Alcalá.

En la base del interés despertado hacia Alcalá, que se puede comprobar leyendo la prensa de entoncesNota5, estuvo sin duda la labor entusiasta, inteligente y arriesgada, de un grupo de alcalaínos a los que vamos a denominar "el grupo 69" porque, formado por personas que desde el 65 hicieron individualmente lo que pudieron por crear opinión favorable dentro y fuera de Alcalá y mover a las instituciones a actuar, fue el 14 de abril de 1969, en una reunión ad hoc, cuando tomaron plena conciencia de la lucha en la que estaban embarcados. Entre estas personas hay que destacar muy particularmente a don José García Saldaña y don Julio Chamorro. Ambos desarrollaron una intensa actividad epistolar ante instituciones, medios de comunicación y particulares; incitaron a manifestaciones, hicieron propuestas de actuación al Ayuntamiento y convocaron numerosas reuniones, entre otras la citada del 14 de abril de 1969. García Saldaña, por otra parte, era y sigue siendo uno de los más destacados investigadores de la historia alcalaína.

La "Universidad Autónoma de Madrid" fue creada por Decreto-Ley de 6 de junio de 1968 y en lo que restaba de ese año, la prensa mantuvo, en general, una clara actitud de apoyo a que ésta se instalase en Alcalá de Henares. Salvador de MadariagaNota6, desde su exilio en Oxford, envió escritos a $A B C$ y a personas alcalaínas en los que abogaba por la Universidad de Alcalá. Su planteamiento del tema estaba claro: 
Leo que van a crear tres universidades más. Estimo [...] que España le debe a Alcalá la Universidad que le quitó. Ha llegado el momento de reparar tan grave errorNota7.

Igualmente podemos decir de universitarios del interior del país, como el Catedrático de la Universidad de Santiago de Compostela don Eugenio Moreno Báez, que pedía:

Que Alcalá vuelva a albergar las Facultades de Derecho y Filosofía, así como las Facultades experimentales [...] dotando así a España de una universidad que podría compararse con las más hermosas del extranjeroNota8.

El Ayuntamiento alcalaíno, movido por la actuación de aquel grupo de personas mencionado y por el propio convencimiento, inició por su parte una serie de actuaciones tendentes a crear también una opinión favorable para la Universidad de Alcalá. Por una parte envió cartas a las autoridades de las Universidades de Coimbra, Oxford, Sorbona y Bolonia, solicitándoles mostraran al Gobierno español su apoyo por la recuperación de esta Universidad que había sido hermana de las citadas en el tiempo y en el prestigio. De ellas se obtuvieron diferentes respuestas, desde el apoyo hasta el silencio, pasando por la elegante inhibición de Oxford. Por otra parte, el Ayuntamiento también propició una reunión de la mayoría de los propietarios de los terrenos que habían sido cedidos en su día para base aérea y que, ahora, si se les cambiaba el destino, tenían todo el derecho legal a exigir su retrovisión.

Esta reunión, celebrada en el verano de 1968, fue muy importante, de hecho y de significado. De hecho porque todos los presentes renunciaron a los derechos que tenían sobre tan extraordinaria extensión de terreno: 
Renunciamos a los posibles derechos de retracto que pueden acogerse con motivo de esta cesión, siempre y cuando los fines de los terrenos se destinen única y exclusivamente a departamentos o servicios universitarios.

y de significado, porque muestra una vez más la identificación de los alcalaínos con la reivindicación de restitución de su pasado universitario, verdadero elemento de solidaridad en la ciudad.

Ya fuera por propio convencimiento ante las facilidades de emplazamiento en Alcalá, o ya fuera porque esas manifestaciones a favor de la ciudad complutense hubieran tenido algún efecto, 1968 terminó con un reconocimiento oficioso de que la Universidad Autónoma se construiría en Alcalá de Henares, cosa que la prensa nacional daba ya como un hecho en Noviembre. El convencimiento se hizo certeza tras el acto de firma de la cesión de los terrenos de la base aérea, por parte del Ministerio del Aire a favor del de Educación, el 20 de diciembre de dicho año.

Dentro de la esperanza que todos estos hechos implicaban para la recuperación universitaria de Alcalá, es interesante resaltar que pocas voces manifestaron la oportunidad de construir aquí un campus que aprovechara la antigua trama urbana universitaria. Ello se justifica, lógicamente por el deseo de aprovechar, ante todo, la oportunidad de los terrenos que se ofrecían. Por ello conviene traer a colación que la idea, no obstante, no estaba falta de inteligentes defensores del retorno, como el Dr. Moreno Báez que, en su segunda intervención periodística a favor de Alcalá decía:

Sería lamentable que se perdiera tan feliz oportunidad de restaurar una de nuestras ciudades más necesitadas de restauración. La nueva Universidad se impregnaría de la gloria que radian las nobles piedras de Alcalá. 
Pero, de todas formas, lo importante es que lo oficioso se hizo oficial con todos los pronunciamientos el 25 de enero de 1969, cuando el Boletín Oficial del Estado publicó la Orden Ministerial de 23 del mismo mes, en la que se decía:

Creada en Madrid una nueva Universidad por Decreto-Ley de 6 de junio de 1968 y afectados al Ministerio de Educación y Ciencia terrenos en Alcalá de Henares, idóneos para el emplazamiento de la misma.

Este Ministerio ha resuelto abrir entre Arquitectos y Sociedades consultoras españolas concurso de anteproyectos para la ordenación de dichos terrenos con arreglo a las bases que a continuación se insertan...

Parecía, pues, que Alcalá de Henares había recuperado su carácter universitario ciento treinta y tres años después de perder su Universidad a favor de Madrid, acogiendo ahora "una universidad de Madrid" en su seno. Era como si la historia universitaria madrileña se reconciliara en el lugar natural de su Alma Mater y, en ese ambiente de celebración, el rector de la Universidad Central (Botella Llusiá), que ya empezaba a gustar de utilizar oficiosamente el impropio nombre de "Complutense" y hacer sus celebraciones más solemnes en el Paraninfo alcalaíno, entregó al alcalde de Alcalá (Félix Huerta en ese momento) la medalla de oro de la Universidad en orden a los méritos contraídos por el Ayuntamiento en la conservación del patrimonio histórico de la misma. Y hay que decir que este homenaje no gustó en Alcalá, donde se hubiera preferido que se le tributara, lógicamente, a la Sociedad de CondueñosNota9.

Sin embargo, la esperanza, que tan oficial y bien fundamentada estaba, duró muy poco. Tan pronto como el 31 de marzo de 1969 un Decreto-Ley determinó la delimitación, ocupación y urbanización de los terrenos para la edificación de la "segunda ciudad universitaria de Madrid" en El Goloso. Inmediatamente se sabría 
que a pesar del eufemismo de "segunda ciudad universitaria de Madrid", el proyecto era el mismo de la Universidad Autónoma o nueva Universidad de Madrid, del que hasta ahora se había tratado. En cualquier caso, esto significaba borrar de un plumazo, y con una disposición de rango superior, la decisión de la instalación en Alcalá.

Es muy difícil saber las razones de esta decisión, que no solamente fue sorprendente entonces, sino que hoy día sigue aún pareciéndolo. Prácticamente toda la prensa, tanto la del régimen como la tímidamente liberal posible, manifestó su incomprensión por la medidaNota10. Madrid planteó la posibilidad de la jugada de grupos especulativos, idea que recogió también Madariaga, como veremos más adelante. De las manifestaciones de un grupo de arquitectos también podría colegirse algo en tal sentidoNota11. La aclaración de los hechos aconsejarían una investigación rigurosa sobre las transferencias de propiedad efectuadas sobre las 100 hectáreas de terreno que se expropiaron en su día en El Goloso, puesto que lo que afectaría a las concesiones de obras, en principio habría de ser indiferente si se efectuaran éstas en un lugar u otro.

Otra línea argumental que se conoce responsabiliza a un sector del Gobierno, encabezado por López Rodó, del secuestro del proyecto de las simples manos del Ministerio de Educación (cuyo titular era Villar Palasí) y su transformación en un tema de Consejo de Ministros, dadas las implicaciones de orden público y de ordenación del territorio que se le atribuían.

Esta segunda línea argumental tiene la virtud de permitir una coherencia explicativa a las manifestaciones oficiales, pocas, que entonces se hicieron, así como a ciertos hechos. En primer lugar, conviene declarar que existe un rumor muy generalizado, que muchos lectores conocen, de que López Rodó inspeccionó la zona en esas fechas e hizo un comentario a sus acompañantes en el sentido del peligro de un núcleo 
universitario en un gran centro de población obrera como era Alcalá y todo el corredor del Henares. En segundo lugar, están las declaraciones que el subsecretario de Educación, Monreal Luque, hizo el 14 de abril de 1969 a una delegación del Ayuntamiento de Alcalá, para explicarles lo sucedido. Según han dejado escrito sus participantesNota12, éste les dijo que la Universidad no se hacía en Alcalá por:

Ser la clave de la industrialización Madrid-Guadalajara.

Se quería hacer en los terrenos de la base aérea un polígono industrial, por el Ministerio de la Vivienda.

Los problemas legales que plantearía el que la segunda universidad de Madrid no estuviera en Madrid.

Decisión del Consejo de Ministros.

En tercer lugar, estuvo la diferente actitud de Villar Palasí y de López Rodó cuando, se vieron sorprendidos en Alcalá, en abril de 1969, por una manifestación de protesta popular por el "robo" de la Universidad de Alcalá. Villar Palasí recibió a la comisión que quería dialogar, López Rodó les increpó y ordenó a la Guardia Civil que interviniera disolviendo a los manifestantes.

De los hechos parece colegirse un caso, tan típico en los regímenes dictatoriales, de acusación dentro de un equipo de una parte a otra de que un hecho determinado "se les ha escapado de las manos" a algún "bienintencionado" poco avezado en los asuntos de poder. Y, efectivamente, si consideramos que justo esos dos años de 1968 y 1969 fueron los de mayor, y más política, contestación estudiantil al régimen, en una situación que todos sabemos era absolutamente incontrolable por el Ministerio de Educación, parece lógico pensar que los miembros más conspicuos del Gabinete (entre los que se contaron Carrero Blanco, López Rodó y Sánchez Arjona) impusieron el criterio de la improcedencia de favorecer con el proyecto universitario de Alcalá la tan temida solidaridad "obreros-estudiantes", que era precisamente el eslogan estelar de la lucha universitaria y política de entonces. De todos es conocida la fama de 
inoperante de Villar Palasí a los efectos del régimen y la sospecha de "reformistas" que tenían ya algunos de los que habían sido sus colaboradores en la elaboración de la "Ley General de Educación". Resulta también revelador que Villar Palasí, en la conversación con la comisión el día de la manifestación, dijera que "en realidad, la Universidad se la habían quitado a él"Nota13.

La respuesta en los meses siguientes a la decisión del Consejo de Ministros muestra la situación de la "idea" de recrear la Universidad de Alcalá: la opinión pública madrileña, en la medida que ésta pudo reflejarse en los medios de comunicación en un régimen sin libertad de prensa, no asumió nunca la reapertura universitaria de Alcalá como forma de recuperar la "memoria histórica universitaria" del centro de España. Es decir, no adoptó como suyo el proyecto universitario de Alcalá.

En la sorpresa por el cambio de emplazamiento, el periódico Madrid y la revista Gaceta Universitaria, entre abril y julio de 1969, asumieron el papel de abogar por la injusticia cometida contra Alcalá y realizaron, aparte de un seguimiento muy detallado de todas las negociaciones y protestas, sendos reportajes sobre todo el asuntoNota14. En ellos se puede observar que las coordenadas de su argumentación son, por una parte, la injusta decepción a la que se ha llevado a una población convencida de que un indudable beneficio iba a repercutir sobre ella, por otra, la sospecha de turbios asuntos especulativos y, por último, el hecho de que puestos a crear una nueva universidad de Madrid, Alcalá era la población con más entidad histórica.

Sólo el grupo de jóvenes arquitectos pertenecientes a la Escuela de Diseño de Madrid, que había elaborado uno de los proyectos para Alcalá, veía claramente que recuperar el carácter universitario de esta ciudad era la oportunidad para Madrid: 
Alcalá era un emplazamiento ideal para crear una Universidad Autónoma, con un completo complejo de residencias, de instalaciones. De lo que se trata es de que la gente pueda estudiar con facilidad; en Madrid esto es difícil por su pésimo urbanismoNota15.

Salvador de Madariaga protestó irónicamente tanto por la sinrazón de haber privado a Alcalá de esta oportunidad, como por la oscuridad de las razones que habían determinado el emplazamiento en El Goloso, a través, fundamentalmente, de sus "Coplas de Alcalaínos", que adquirieron su máxima difusión al ser publicadas en $\mathrm{ABC}$ :

Que a Cisneros y a Cervantes quitan lo que dieron antes, Albalá contra albalá. ¿Por qué se deja Alcalá?

Que a Cervantes y a Cisneros por hectáreas o dineros, El Goloso vencer ha, ¿por qué se deja Alcalá?

Que sería vergonzoso que venciera ese goloso en lucha en que tanto va, ¿por qué se deja Alcalá?

Que si en la villa del oso se ha escondido ese goloso, el Buscón lo encontrará, 
¿por qué se deja Alcalá?

\author{
Si las razones que da \\ para los alcalaínos \\ son coplas de Calaínos, \\ ¿por qué se deja Alcalá?
}

Y si la Universidad,

en vez de una disciplina,

va a ser una golosina,

¿por qué se deja Alcalá?

\title{
Y pues Alcalá se deja
}

cuando en ello tanto va,

sobre qué Alcalá se queja?

Mal se queja quien se deja,

¿por qué se queja Alcalá?

Lo que estaba en la misma línea de su solicitud en $A B C$, unas semanas antes, de explicación sobre el asunto:

Tan evidente es el derecho natural de Alcalá, tan inculta e injusta es la decisión de trasladarla [la universidad] a Madrid, que antaño se hizo en hora de triste recordación. [Por ello] el pueblo español desearía saber qué razones hay de tan formidable fuerza que hayan vencido a Cisneros y a Cervantes en el ánimo de los gobernantes: y si tan fuertes son, ¿por qué no se publican? Y si no se publican, ¿quién es El Goloso?Nota16 
Las anteriores son muestras de la formación de un pequeño estado de opinión favorable a la recuperación universitaria de Alcalá entre algunos sectores reducidos que, si bien es difícil calibrar si tuvo efecto para el futuro, se puede calificar como el momento histórico en el que termina la etapa, iniciada en 1836, en la que la tradición universitaria de Alcalá "se esta olvidando".

Ahora bien, los medios de comunicación madrileños, y por tanto nacionales, se hicieron eco del problema de Alcalá en ese verano de 1969 gracias a que, como dijimos antes, un grupo de habitantes de Alcalá desarrollaron una serie de pequeñas, pero inteligentes y arriesgadas para la época, acciones de protesta, con las que no sólo mantuvieron vivo el problema, sino que reafirmaron el carácter ciudadano y suprapolítico de esta reivindicación, y obligaron a la realización de ambiguas promesas de no olvidar universitariamente a Alcalá.

Ese es el grupo que hemos denominado grupo 69, formado por unas cuarenta personas que se reunieron, a instancias de García Saldaña y Chamorro, el día 14 de abril de 1969Nota17. De esta reunión surgieron una serie de compromisos de actuación, colectivos e individuales, que se plasmaron en el mantenimiento de un permanente estado de opinión contra la decisión de traslado a El Goloso y la realización de un conjunto de acciones de negociación al respecto.

Entre esas actuaciones destacó la labor de "agitación gráfica", que cubrió los monumentos de la ciudad con carteles que llamaban a la movilización ciudadana. Así, a Cisneros se le colocó un "¡Alcalaínos recuperad mi Universidad!", a Cervantes "¡Alcalaínos!, como Fuenteovejuna todos a una, para que vuelva a Alcalá su Universidad" y "¡Visitantes!, en 1836 nos despojaron la Universidad, hoy pretenden hacerlo nuevamente, ¡no lo permitáis!"Nota2 de VA. Y, sobre todo, incitaron al Ayuntamiento (cogido entre la lealtad fascista al régimen, de unos miembros que pertenecían todavía en su mayoría a la vieja guardia de la Guerra Civil, y la lealtad a 
la reivindicación histórica, consustancial a su condición de alcalaínos), a que hiciera gestiones ante las autoridades nacionales y provinciales, pidiendo explicaciones.

Esta "confabulación" de un grupo de ciudadanos a favor de la Universidad, creemos que tuvo una indudable trascendencia política para el futuro de la ciudad, aparte del significado universitario. Sirvió como elemento de concienciación contra el régimen de personas que hasta ese momento habían sido adictas al mismo, con cargos incluso en la organización local del Movimiento - de los que dimitieron algunos en ese momento-, y sirvió para que se encontraran personas del antiguo régimen con otras que militaban en grupos clandestinos de izquierdas. Algunas de estas últimas, en diferentes grupos políticos (como Arsenio Lope Huerta, Heliodoro Ceballos, Francisco González Adrada), ocuparían cargos de responsabilidad municipal durante la democracia y colaboraron entonces a que el tema de la recuperación universitaria de Alcalá fuera un asunto de coincidencia política en la ciudad.

Como síntesis de lo que para el sentimiento alcalaíno significó el fiasco de la creación y cierre, en el plazo de tres meses, de su nueva universidad, se puede señalar la esquela mortuoria que algún miembro del grupo 69 hizo imprimir y exhibir en muchos establecimientos alcalaínos y que decía:

UNIVERSIDAD AUTÓNOMA COMPLUTENSE. Falleció en Madrid el 15 de abril de 1969. D.E.P. Tus alcalaínos no te olvidan. Sus restos serán trasladados, desde su emplazamiento en esta ciudad, a los terrenos de El Goloso (Madrid). Esperemos que a quienes nos aflige tan sensible pérdida nos consuele, un día, ver su resurrección transformada en grandioso Instituto Politécnico.Nota3 de VA

Es decir, por un lado, amarga resignación pero, por otro, manifestación de no olvidar, y esperanza. Lo de Instituto Politécnico muestra la ironía ante una de las tantas promesas que, en el curso de la solicitud de explicaciones, hizo el Ministerio 
de Educación para acallar la protesta. Otras fueron más genéricas, como la carta que envió el ministro Villar Palasí al alcalde diciendo lacónicamente que:

No hemos desistido de la posibilidad de instalar en esa ciudad algunos de los centros facultativos de la Universidad Autónoma de Madrid (29 de abril de 1969).

Sin duda, lo que había detrás de todo esto, como los hechos demostraron más adelante, era que el Ministerio de Educación disponía en Alcalá, gracias a la generosidad de los alcalaínos para con su pasado histórico, de 240 hectáreas de terreno que siempre quiso conservar para cualquier proyecto educativo.

CONTINUARÁ...

En el próximo número: 1972-1974: nueva apertura y cierre de una universidad en Alcalá

\section{NOTAS}

NOTA2: Datos de Análisis de Alcalá de Henares. Instituto de Estudios de la Administración Local. Madrid. 1948.

NOTA3: Datos del Padrón de Vecinos de 1975.

NOTA4: Datos de "Estudio Socio-Económico de Alcalá".

NOTA5: Ver ABC: 26-11-68,30-11-69, 22-12-68, 28-12-68, 26-1-69, 8-3-69, 2-4-69;

Gaceta Universitaria: 15-1-69; Arriba: 2-4-69; Ya: 2-4-69, 3-4-69. 
NOTA6: Considérese la significación del hecho, no tanto por el peso intelectual del personaje, sino también por lo que tiene de cierre de la etapa de aquel "liberalismo" ilustrado y reformista, que había liquidado en su día la Universidad de Alcalá. Si alguna figura intelectual podía representar el liberalismo histórico español en los años del exilio era, precisamente, Salvador de Madariaga.

NOTA7: ABC, 3 de julio de 1968.

NOTA8: MORENO BÁEZ, E: En la sección de "Opiniones ajenas, polémicas, cartas, puntualizaciones, comentarios". ABC, 14 de julio de 1968.

NOTA9: La Universidad de Alcalá, al ser trasladada a Madrid por Real Decreto de 1836 conservó sus archivos (que más adelante, no obstante, pasaron al Archivo Histórico Nacional), sus ejecutorias y su escudo, pero cambió su nombre por el de "Universidad Central". La Ley de Ordenación Universitaria de 1943 le suprimió el calificativo de Central y pasó a llamarse "Universidad de Madrid" e insertó en su escudo el lema "Universitas Complutensis, Universidad de Madrid". En 1970, al redactar su anteproyecto de "estatutos provisionales", instauró como nuevo nombre el de "Universidad Complutense de Madrid", sin duda para distinguirse de la Autónoma y recogiendo el ambiente de "recuperación de señas de identidad " al que nos hemos referido. El nombre de "Complutense" (aberración geográfica e histórica para una universidad de la ciudad de Madrid), al estar recogido en unos Estatutos que fueron sólo "provisionales" hasta la Ley de Reforma Universitaria de 1983, era, por tanto, sólo oficioso. Pero desgraciadamente, tras la Ley de Reforma Universitaria la aprobación, por Ley, de los nuevos Estatutos de la Universidad de Madrid, éstos conservaron el nombre de "Complutense". Teniendo en cuenta que existía ya desde 1977 la nueva Universidad de Alcalá, la responsabilidad de haber perdido la ocasión de poner en orden las cosas afecta, según mi criterio, a todas las partesNota1 de VA . Por cierto que, en esa historia de despropósitos sobre la simbología universitaria de 
Alcalá, la Universidad Autónoma de Madrid, una vez que se tomó la decisión de instalarla en El Goloso, sus autoridades propusieron que se llamara "Cardenal Cisneros", lo que se hacía, no cabe duda, por la vinculación del mismo a su obra universitaria, cual fue la Universidad de Alcalá. Por suerte, la intención no llegó a prosperar y Alcalá al menos sigue utilizando, informalmente, pero con todo derecho, el calificativo de "Universidad cisneriana" cuando desea referirse a las edificaciones históricas de la misma.

NOTA10: La única nota discordante, y que merece la pena recordar por lo ridículo de la argumentación -muy en la línea, no obstante, de un fascismo que andaba en retirada- fue la de un articulista de Arriba, OCTAVIO RONCERO, que justificaba de esta manera el abandono de Alcalá por El Goloso: "Alcalá está demasiado unida a la clase dominante española de siglos y es bueno que un nombre popular, venido del pueblo, con sabor proletario, llegue hasta la universidad. Es casi una afirmación de un país en marcha que va hacia delante, y que las ataduras de las castas y de los "hijitos de papá han dejado de preocuparle"" (Arriba: "Nombre proletario para una universidad", 17 de junio de 1969). Es curiosa esa coincidencia de lenguaje con el empleado por algunos de los que hoy se llaman de izquierdas, cuando se refieren a los asuntos universitarios.

NOTA11: Gaceta Universitaria, num. 125, 2ª quincena, julio, 1969.

NOTA12: SANZ, Aseia y GARCÍA GUTIÉRREZ, Javier: "La lucha por la Universidad. Historia próxima de un retorno". Puerta de Madrid. Especial Retorno de la Universidad, 1975.

NOTA13: Gaceta Universitaria, núm. 125, 2ª quincena, julio, 1969. 
NOTA14: En los escaparates de algunas tiendas de Alcalá aparecieron durante el mes de mayo carteles que decían lo siguiente: Diario Madrid: Habla de tu pueblo, ¡Léelo!

NOTA15: Gaceta Universitaria, núm. 125, 2ª quincena, julio, 1969.

NOTA16: DE MADARIAGA, Salvador. Carta en ABC. 25 de mayo de 1969.

NOTA17: La reunión se celebró a las 10 de la noche en el mesón "Las Cuadras de Rocinante", y asistieron unas 40 personas. La convocatoria se hizo a instancias de José García Saldaña y Julio Chamorro, contando con la arriesgada colaboración del propietario del negocio don José Quijada.

NOTA1 de Vivat Academia: Por aquel entonces ya era rector de la Universidad de Alcalá Manuel Gala.

NOTA2 de Vivat Academia: Las negritas son nuestras.

NOTA3 de Vivat Academia: ¿Profecía? Resulta curioso que el rector Gala haya hecho realidad las promesas del gobierno fascista de entonces. 